\title{
ACOUSTIC INTERACTIONS IN ARRAYS OF SPHERICAL ELASTIC SHELLS
}

\author{
C. L. Scandrett and D. R. Canright \\ Mathematics Dept., Code 53 \\ Naval Postgraduate School \\ Monterey, CA 93943
}

\begin{abstract}
:
The acoustical performance of a submerged linear array of spherical transducers is examined by combining the T-Matrix method of solving for multiple acoustic interactions among separate bodies with a model for the transducers as thin spherical elastic shells. This approach solves the fully coupled problem of the response of the array to internal forcing. The results show that the assumptions giving rise to the Chebyshev criteria for optimal arrays of point sources appear to apply well even for large spheres at low frequencies. However, at frequencies near or above the lowest resonant frequency the directional pattern may be degraded, depending on the material of the shells.
\end{abstract}




\section{Introduction}

The determination of the acoustical field due to several separate radiating and/or scattering bodies is a problem of considerable practical importance, and has been extensively studied. The present work concerns the interactions of an array of spherical radiators, considered to be elastic shells, in an unbounded medium.

While there has been much related work on scattering from spheres and on radiation from different geometries, e.g., cylindrical radiators or flat disks, the previous work on multiple spherical radiators is not extensive. Karnovskii $(1941,1956)$ examined the effect on the resistive portion of the impedance of a spherical radiator due to the presence of other radiators in an array, under the assumptions that the wavelength is much larger than the radiator, and the surface velocity of each radiator is specified. New and Eisler (1972) applied a more accurate method, with no restriction on wavelength, to the problem of a pulsing sphere near a rigid sphere. Their method of accounting for multiple acoustic interactions (equivalent to that used by Marnevskaya $(1969,1970)$ for scattering from two spheres) involves decomposing the total acoustic field into waves radiated from each sphere, which in turn are expanded in terms of spherical waves. The boundary conditions on each sphere are satisfied by translating the coordinate origin of each other sphere to that of the first by means of an "addition theorem" for spherical waves (Friedman and Russek, 1954). We use the same method here.

Waterman (1969) developed this method more generally for scattering from a body of arbitrary shape. This was extended to apply to several bodies by Peterson and Ström (1974), and also by Varadan and Varadan (1981). This technique of finding multiple acoustic interactions is commonly referred to as the "T-matrix" method.

Thompson (1977) extended the work of New and Eisler by considering different velocity distributions on the two spheres: both spheres pulsing or both oscillating, in or out of phase. Reese and Thompson (1981) further considered the next higher axisymmetric mode of vibration $(n=2)$, and Thompson and Reese (1983) considered the combination of one pulsing and one oscillating sphere.

In all of the aforementioned work, the surface velocities on the spheres are assumed to be known a priori, i.e., the spheres are treated as perfect velocity transducers. In this way the above work is idealized in the sense that the effects of fluid loading on the structures are not taken into account.

Much work has been done on scattering by elastic bodies which does account for the coupling between the acoustic field and the elastic deformation. Here, we mention only a few relevant papers. Junger (1952) examined the scattering of plane waves by a thin spherical shell. Forced vibrations of a spheroidal shell, and the resulting acoustic field, were considered by Yen and DiMaggio (1967). Böstrom (1980) developed a method, closely related to the T-Matrix method, for acoustic scattering from an elastic body of arbitrary shape. In his paper, he gives numerical results for spheroid and "peanut" shaped scatterers. Other researchers have employed the T-Matrix method to treat scattering from bodies of more complicated geometry, e.g., Peterson et al. (1980). Seybert et al. (1988) have recently investigated radiation by a forced thick spherical shell by a boundary-element 
method. In all of the above mentioned works dealing with elastic radiators, it should be noted that results are given only for a single radiator rather than for an array.

Here we consider an array made up of a small number of radiators in an unbounded medium. The method used combines the "T-Matrix" method for multiple acoustic interactions with a mechanical model of each radiator, treated as a thin elastic spherical shell with specified internal forcing. This approach solves the coupled problem for the acoustic field (including multiple-scattering effects) and the motion of the elastic shells. While we consider the radiation problem, the method is equally applicable to the combined radiation-scattering problem, by the inclusion of an externally applied incident acoustic field.

The linear array considered is comprised of three identical spherical shells, which are forced by a uniform pressure along the inside of each shell, thereby exciting the lowest (pulsing) mode. For comparison, we solve the same problem for spheres which have their surface velocities specified. We find that the theoretical predictions for an array of point sources with Chebyshev spacing and shading apply well to the array considered when the frequency of excitation is less than the lowest resonance frequency of a single spherical shell in water. This corresponds to values of roughly $k a=1.0$ for the case of steel shells, where $a$ is the radius of the shells, and $k=\omega / c_{f}$. For frequencies above the lowest resonance frequency the theoretical predictions can also be very good depending upon the material properties of the scatterers involved and whether or not they are being excited at resonance.

\section{Method}

The acoustic field $p$ in an unbounded medium containing a finite number of bodies $(M)$ can be written as the sum of radiated pressures from each body $p_{j}$ and the incident pressure $p_{0}$ (if any):

$$
p(\mathbf{x})=p_{0}(\mathbf{x})+\sum_{j=1}^{M} p_{j}(\mathbf{x})
$$

where $p$ is the total pressure (complex), $\mathbf{x}$ is vector position and all variables are assumed to vary harmonically in time as $e^{-i \omega t}$, with angular frequency $\omega$. Hereafter we consider only the radiation problem for driven transducers, i.e., we assume there is no incident pressure.

Each of the radiated pressures $p_{j}$ can be expressed as a series whose terms $\Phi_{m n}$ form a complete set of separable radiating solutions to the reduced Helmholtz equation in a particular coordinate system. Here we use spherical harmonics, but one could instead use spheroidal or ellipsoidal harmonics. Then:

$$
p_{j}\left(r_{j}, \theta_{j}, \phi_{j}\right)=\sum_{n=0}^{\infty} \sum_{m=-n}^{n} A_{j m n} h_{n}^{(1)}\left(k r_{j}\right) Y_{n}^{m}\left(\theta_{j}, \phi_{j}\right)
$$

Here $\left(r_{j}, \theta_{j}, \phi_{j}\right)$ are spherical coordinates relative to the center of radiator $j, A_{j m n}$ is the 
amplitude of the corresponding spherical wave, which is the product of the spherical Hankel function $h_{n}^{(1)}\left(k r_{j}\right)(k=\omega / c$ is the wavenumber) and the surface spherical harmonic $Y_{n}^{m}\left(\theta_{j}, \phi_{j}\right)=P_{n}^{m}\left(\cos \left(\theta_{j}\right)\right) e^{i m \phi_{j}}$, where $P_{n}^{m}$ is the associated Legendre function. For arbitrarily shaped bodies, this expansion is only valid exterior to all the spheres circumscribed about each body (Waterman, 1969), but since we assume spherical radiators, the expansion is valid throughout the medium.

If the normal velocities $u_{j}$ at the surface of each spherical radiator are known a priori then solving the multiple-scattering problem amounts to satisfying the boundary conditions on the surface of each sphere:

$$
\left.\frac{\partial p}{\partial r_{j}}\right|_{r_{j}=a_{j}}=(i \rho c k) u_{j} \quad, \quad j=1,2, \ldots M
$$

This is facilitated by the "addition theorem" that expresses a spherical wave relative to one origin as a series of spherical waves relative to another origin (Friedman and Russek, 1954). We use the form of the addition theorem given by New and Eisler (1972), based on the formulation of Sack (1964). Thus the complete acoustic field $p$ due to all the radiators can be represented in terms of spherical waves relative to any one particular radiator $(j)$ :

$$
p\left(r_{j}, \theta_{j}, \phi_{j}\right)=\sum_{n=0}^{\infty} \sum_{m=-n}^{n}\left[A_{j m n} h_{n}^{(1)}\left(k r_{j}\right)+\left(\sum_{k=1, k \neq j}^{M} B_{j k m n}\right) j_{n}\left(k r_{j}\right)\right] Y_{n}^{m}\left(\theta_{j}, \phi_{j}\right)
$$

where $j_{n}\left(k r_{j}\right)$ is the spherical Bessel function of the first kind, and the coefficients $B_{j k m n}$ are given by the addition theorem of the form

$$
\begin{aligned}
B_{j k m n} & =\sum_{\nu=0}^{\infty} \sum_{\mu=-\nu}^{\nu} T_{j k m n \mu \nu} A_{k \mu \nu} \\
T_{j k m n \mu \nu} & =\sum_{s=|\nu-n|}^{\nu+n} a(n, s, \nu, m, \mu) h_{s}^{(1)}\left(k r_{j k}\right) Y_{s}^{\mu-m}\left(\theta_{j k}, \phi_{j k}\right)
\end{aligned}
$$

where the summation over $s$ is in increments of 2 , the coordinates $\left(r_{j k}, \theta_{j k}, \phi_{j k}\right)$ give the center of sphere $j$ relative to that of sphere $k$, and the translation theorem coefficients $a(n, s, \nu, m, \mu)$ are given in New and Eisler (1972). This representation applies everywhere within any sphere centered on radiator $j$ that excludes all the other radiators, and in particular applies on the surface of radiator $j$.

The normal derivative of pressure at the surface of radiator $j$ is found from (4) by replacing the Hankel and Bessel functions $h_{n}^{(1)}\left(k r_{j}\right), j_{n}\left(k r_{j}\right)$ by their derivatives, evaluated at $k a_{j}$, where $a_{j}$ is the radius of sphere $j$. This gives a series in terms of the surface harmonics $Y_{n}^{m}$. The surface normal velocities $u_{j}$ are likewise expanded in terms of surface harmonics, with coefficients $U_{j m n}$. Due to the orthogonality of these functions, the coefficients of $Y_{n}^{m}$ on each side of the series representation of equation (3) must be equal for each sphere. In this way, the boundary conditions for the complete set of spheres reduce to a matrix equation of the form

$$
\underline{\underline{Y_{a}}} \underline{A}=\underline{U}
$$


Here, the vectors $\underline{A}$ and $\underline{U}$ represent respectively the coefficients $A_{j m n}$ for the radiated pressure field (as in Eq. 2) and the coefficients $U_{j m n}$ in the expansion of the normal surface velocity, while $\underline{\underline{Y_{a}}}$ is an acoustic admittance matrix that accounts for all the multiple scattering. Given the surface velocities on each sphere, one can solve for the acoustic field $(\underline{A})$ by a matrix multiplication of equation $(6)$ by the acoustic impedance matrix $\underline{\underline{Z_{a}}}=\left(\underline{\underline{Y_{a}}}\right)^{-1}$.

The above approach assumes the radiators are ideal velocity transducers in that their surface velocity is entirely independent of the acoustic loading. Instead, the radiators can be treated more realistically as thin spherical elastic shells, with internal drivers that apply a known force. The resulting motion of the shells is then due to a combination of internal forcing and acoustic loading. This fully accounts for the acoustic coupling of the fluid/structure interactions.

The motion of a thin elastic spherical shell subject to harmonic forcing is well known. We use the thin shell equations of motion which are equivalent to the formulation given by Junger and Feit (1972) with $\beta=0$. The surface normal motion can be represented in terms of spherical harmonics, each corresponding to two normal modes of vibration of the shell (or one mode for $n=0$ ). Associated with each normal mode of vibration of the shell is a mechanical impedance. Utilizing this impedance, the coupled fluid/structure equations reduce to a set of linear algebraic equations (as above):

$$
\underline{F}-\underline{A}=\underline{\underline{Z_{m}}} \underline{U}
$$

where the vector $\underline{F}$ represents the coefficients $F_{j m n}$ for the internal forcing, and the matrix $\underline{\underline{Z_{m}}}$ is the mechanical impedance matrix, which is diagonal. (The pressure term is negative because the pressure acts inward, not outward.)

The modal impedances of the $j^{t h}$ radiator along the diagonal of $\underline{\underline{Z_{m}}}$ are given by:

$$
Z_{j m n}=-\frac{a_{j}^{2}\left(1-\nu^{2}\right)}{E h}\left\{\frac{\Omega^{2}-\nu-\lambda_{n}+1}{\left(\Omega^{2}-\nu-\lambda_{n}+1\right)\left(\Omega^{2}-2(1+\nu)\right)-\lambda_{n}(1+\nu)^{2}}\right\}
$$

where the scaled frequency is $\Omega^{2}=a_{j}^{2} \omega^{2}\left(1-\nu^{2}\right) \rho / E, a_{j}$ is the sphere radius, $h$ is the shell thickness, $\rho$ is the density, $E$ is Young's modulus, $\nu$ is Poisson's ratio, and $\lambda_{n} \equiv n(n+1)$. Note that, in this thin-shell approximation, for a given number $n$ of nodes, all the surface harmonics corresponding to different azimuthal dependence numbers $m$ have the same impedances.

Combining equations (6) and (7) produces a matrix equation which can be solved for the surface motion directly in terms of the forcing:

$$
\underline{U}=\left(\underline{\underline{Z_{m}}}+\underline{\underline{Z_{a}}}\right)^{-1} \underline{F}
$$

This demonstrates that for the coupled problem, the total effective impedance is the sum of the acoustic impedance and the mechanical impedance. (This simplicity is a result of the spherical geometry of each transducer.) The velocities then yield the acoustic field through equation (6). 
The far-field radiation is more conveniently expressed in terms of spherical waves relative to a single system coordinate origin (rather than by equations 1 and 2). This entails applying the addition theorem again, in a slightly different form, to express the radiation from each radiator in terms of the system origin. Using the index zero to indicate the system origin, the appropriate addition theorem is:

$$
\begin{aligned}
p\left(r_{0}, \theta_{0}, \phi_{0}\right)= & \sum_{j=1}^{M} \sum_{n=0}^{\infty} \sum_{m=-n}^{n} A_{j} m n\left\{\sum_{\nu=0}^{\infty} \sum_{\mu=-\nu}^{\nu} j_{\nu}\left(k r_{0 k}\right) Y_{\nu}^{\mu}\left(\theta_{0 k}, \phi_{0 k}\right)[\right. \\
& \left.\left.\sum_{s=|n-\nu|}^{n+\nu} a(\nu, s, n, \mu, m) h_{s}^{(1)}\left(k r_{0}\right) Y_{s}^{m-\mu}\left(\theta_{0}, \phi_{0}\right)\right]\right\}
\end{aligned}
$$

where the summation over $s$ is in increments of 2 and the notation follows that of equations (4) and (5). This representation applies anywhere outside a sphere centered at the origin that contains all the radiators.

In practice, the infinite sums over mode numbers ( $n$ and $\nu$ ) must be truncated at some particular number, say $N$. (Then in equation 10, the sum over $\nu$ would go up to $2 N$ for consistency.) Also, if the array is linear and the forcing is axisymmetric about this line, only axisymmetric modes $(m=0)$ will be excited, eliminating all sums over $m$ and $\mu$.

To summarize our method, given the geometry of the array, the properties of the medium, and the operating frequency, the acoustic impedance matrix $\underline{Z_{a}}$ can be calculated, up to the chosen number of modes $N$, through the addition theorem. From the material properties and thickness of each shell, the mechanical impedance matrix $Z_{m}$ can be found. The sum of the two gives the total impedance matrix, which can be used to determine the surface motion of each transducer, given a prescribed internal forcing. The motion in turn determines the acoustic field, which in the far field is given by a second application of the addition theorem.

\section{Results}

Numerical results are presented which: 1) demonstrate that the spherical addition theorems have been correctly applied; 2) illustrate the response of a small linear array of radiating spherical shells given Chebyshev shading; and, 3) examine convergence properties of the truncated infinite series of spherical harmonics over a specified frequency window.

To confirm that the translation formulas for the spherical harmonics have been correctly applied, results reported in New and Eisler (1972) were reproduced for the particular problem of two submerged spheres, one of which is pulsing harmonically, while the other acts like an acoustically hard obstacle. Fig. 1 displays the reactance and resistance terms of the acoustic impedance which have been scaled by their corresponding values obtained from a single harmonically pulsing sphere. Variations about unity represent the extent to which the reactance and resistance of the pulsing sphere are affected by the presence of 
the second sphere. As one would expect, and as can be seen from Fig 1, the interaction effect is mitigated as the scaled distance $(k r)$ between spheres is increased.

Note that there is excellent agreement between the present calculations, using four spherical harmonics, with those of New and Eisler (using eleven). Fig. 2 plots the corresponding far field pattern of the present code against that of New and Eisler once again showing very good agreement. The far field results of Fig. 2 have been scaled by the magnitude of the far field produced from a single pulsing sphere.

For the array problem, the three spheres are centered at $(0,0,0)$ and $(0,0, \pm D)$. The material properties (assuming steel) and dimensions of the shells used are as follows:

Radius, $a=1.0 \mathrm{~m}$

Thickness, $h=.01 \mathrm{~m}$

Young's Modulus, $E=2.07 \times 10^{11} \mathrm{x}(1+i \eta) N / m^{2}$

Structural Loss Factor, $\eta=0.0$ or 0.1

Poisson's Ratio, $\nu=0.3$

Shell Density, $\rho_{s}=7669 \mathrm{Kg} / \mathrm{m}^{3}$

Fluid Density, $\rho_{f}=1000 \mathrm{Kg} / \mathrm{m}^{3}$

Fluid Sound Speed, $c_{f}=1524 \mathrm{~m} / \mathrm{sec}$

Scaled Center to Center Spacing, $k D=\pi$ or $D / \lambda=1 / 2$.

The Chebyshev criteria for an array of point sources suggests that one can control the magnitude of the side lobes relative to the main lobe by adjusting the amplitudes of the forcing functions applied to the surfaces of the spheres (Dolph, 1946). Here the spheres on either side of the center sphere are given relative amplitudes $11 / 18^{\text {ths }}$ that of the center sphere (which is subject to a uniform pressure of $1 \mu \mathrm{Pa}$ ) to give a $20 \mathrm{~dB}$ drop. Given the half wavelength spacing of the spheres, and the foregoing amplitudes, the main lobe should appear at $\theta=90$ degrees, with side lobes (magnitudes $20 \mathrm{~dB}$ less than the center lobe) at $\theta=0$ and 180 degrees.

In the results that follow, the source level $(S L)$ of the array of radiators is defined by

$$
S L=10 \log _{10}\left[\lim _{r \rightarrow \infty}\left(\frac{k r p(r, 0, \pi / 2)}{1 \mu P a}\right)^{2}\right] .
$$

The above source level is in practice found by taking the far field pressure, and scaling it to $1 \mu P a$. Far field results are scaled in an equivalent fashion.

Fig. 3 displays the radiation pattern at $k a=1.13$, which is near the first resonance frequency for a submerged spherical shell. The convergence of the truncated series is shown by varying the number $N$ of spherical harmonics retained. It can readily be seen that the series solution for the pressure has converged with the inclusion of 6 terms. In subsequent results 11 harmonics are used to obviate convergence concerns.

Fig. 4 compares the far field results at four different frequencies; $k a=0.9, k a=1.0$, $k a=1.25$, and $k a=1.5$. It can be seen that for the low frequency cases the Chebyshev array produces a result matching that of an idealized Chebyshev array, in that the nulls appear where they should, and the pressure at 90 degrees is $20 \mathrm{~dB}$ greater than the pressures at 0 and 180 degrees. The trend, however, as the frequency is increased, is that the 
response smooths out. The nulls have nearly vanished, and the far field appears to be tending towards a uniform far field pressure. The conclusion is that at low frequencies Chebyshev optimality is preserved, while at higher frequencies optimality is destroyed.

To examine the effect of varying the frequency on the response of the array, Fig. 5 graphs the $S L$ calculation over the frequency range $k a=0.01$ to $k a=1.5$. Three curves are shown which correspond to arrays of spheres with prescribed surface velocities; as well as damped $(\eta=0.1)$, and undamped $(\eta=0.0)$ thin-walled spherical shells. The response curves appear to be relatively smooth in all cases below frequencies about $k a=1.0$. The response curve of the array of spheres with prescribed surface velocities is in fact smooth throughout the frequency window, while that of the spherical shells without damping has a prominent dip at $k a=1.13$, at which point the $S L$ drops by almost $15 d B$. A second less significant spike appears at $k a=1.44$. The introduction of the mechanical damping has the effect of smoothing out the spikes at these anomolous frequencies, especially at $k a=1.44$ where the spike completely disappears in the damped response.

To more carefully analyze the array response about $k a=1.13$, Fig. 6 plots the directional responses of the array from $k a=1.11$ to $k a=1.14$. At $k a=1.13$ it is seen that the peak at $\theta=90$ degrees dips, leaving lobes at $\theta=60$ and 120 degrees. This frequency is slightly less than the lowest eigenfrequency of a single submerged spherical shell, which is at about $k a=1.15$. The response at $k a=1.13$ would appear resonable in light of the fact that the $n=2$ spherical harmonic takes on its most negative value at $\theta=90$ degrees as it has a $\theta$ dependence similar to $\cos (2 \theta)$. The spike at $k a=1.44$ is likewise very close to the eigenfrequency of the $n=3$ spherical harmonic.

To further support the contention that the spikes occuring in the shell response of fig. 5 are due to resonance effects fig. 7 is included. Fig. 7 plots directional response curves for the array of spheres with specified surface velocities at identical frequencies to those displayed in fig. 4 for the steel shells. Notice that at each frequency of fig. 7 , the response changes little, and in fact is very close to the predicted values for an idealized Chebyshev array.

Figs. 8 and 9 are equivalent to figs. 5 and 4 except that aluminum spherical shells are used. All dimensions are the same as for the steel shells. The pertinent material properties used for aluminum are:

Young's Modulus, $E=7.10 x 10^{10} x(1+i \eta) N / m^{2}$

Poisson's Ratio, $\nu=0.33$

Shell Density, $\rho_{s}=2700 \mathrm{Kg} / \mathrm{m}^{3}$.

As is seen from examination of fig. 8, a spike in the source level response is found at $k a=.76$ which agrees with the eigenfrequency of the $n=2$ spherical harmonic. The spike is not as dramatic as in the case of the steel shell, and in fact upon viewing the far field response at this frequency, the $n=2$ harmonic is only slightly excited, causing only a slight deformation of the far field response. To show that the aluminum shells act more like an idealized Chebyshev array, fig 9 plots four far field curves above $k a=.76$. It can be seen that the idealized shape is roughly maintained. The above evidence suggests that for at least certain materials, excitation above resonance does not necessarily degrade the idealized Chebyshev output of the array.

Finally, figure 10 compares the directional patterns due to arrays of point sources, 
spheres with prescribed surface velocities, steel shells, and aluminum shells, for $k a=1.0$. This shows that the main geometric effect of using large spheres rather than point sources is that the nulls at 30 and 120 degrees are less pronounced for large spheres. The effects of differing materials and driving (force vs. velocity) primarily give different overall levels of output, with similar directional dependence, at this frequency.

In conclusion, more work needs to be done in exploring the interactions of radiating arrays and the present methodology appears to provide a versatile tool with which this can be done. One advantage of the present technique over many of the methods currently used, is that in the performance of the array at a particular frequency, the amount of excitation of each eigenmode of the shells can be seen explicitly. This cannot be done easily if the problem has been solved numerically via finite difference, finite element, or boundary element methods. Furthermore, the acoustic impedance is found independently of the mechanical impedance, which breaks the coupled problem into two simpler problems. Thus in exploring, say, the effects of the radiator material and shell thickness for a particular array geometry, the acoustic impedance need be computed only once, and then coupled with the mechanical impedance of each type of radiator. In future work we plan to use this approach with more complicated transducer models. 


\section{Bibliography}

A. Boström, J. Acous. Soc. Amer. 67, 390 (1980).

C. L. Dolph, Proc. Inst. Radio Engr. 34, 335 (1946).

B. Friedman and J. Russek, Quart. of Appl. Math. 12, 13 (1954).

M. C. Junger and D. Feit, Sound, Structures, and Their Interaction (MIT Press, Cambridge, MA, 1972).

M. C. Junger, J. Acous. Soc. Amer. 24, 366 (1952).

M. I. Karnovskii, Soviet Phys.- Acoustics 2, 280 (1956).

M. I. Karnovskii, Doklady Akad. Nauk SSSR 32, 40 (1941).

L. A. Marnevskaya, Soviet Phys.- Acoustics 14, 356 (1969).

L. A. Marnevskaya, Soviet Phys.- Acoustics 15, 499 (1970).

R. New and T. J. Eisler, J. Sound Vib. 22, 1 (1972).

B. Peterson and S. Ström, J. Acoust. Soc. Am. 56, 771 (1974).

B. Peterson, V. V. Varadan, and V. K. Varadan, J. Acoust. Soc. Am. 68, 673 (1980).

J. M. Reese and W. Thompson, Jr., J. Acoust. Soc. Am. 69, 1587 (1981).

R. A. Sack, J. Math. Phys. 5, 252 (1964).

A. F. Seybert, T. W. Wu, and X. F. Wu, J. Acoust. Soc. Am. 84, 1906 (1988).

W. Thompson, Jr., J. Acoust. Soc. Am. 62, 8 (1977).

W. Thompson, Jr. and J. M. Reese, J. Acoust. Soc. Am. 74, 1048 (1983).

V. V. Varadan and V. K. Varadan, J. Acoust. Soc. Am. 70, 213 (1981).

P. C. Waterman, J. Acous. Soc. Amer. 45, 1417 (1969). 
T. Yen and F. DiMaggio, J. Acous. Soc. Amer. 41, 618 (1967). 


\section{Figure Captions}

Figure 1. Resistance and reactance for one pulsing sphere (velocity prescribed) near a hard sphere, normalized by the value in the absence of the second sphere, as a function of the scaled distance $k r$ between the sphere centers.

Figure 2. Normalized far field directional dependence; same configuration and comparison as in Figure 1, for $k r=3$.

Figure 3. Directional pattern for Chebyshev array of three steel spherical shell radiators, near a resonance $(k a=1.13)$, calculated using varying numbers of harmonics $N$. (Grid circle intervals are $20 d B$.)

Figure 4. Frequency dependence of directional pattern of array (as in Figure 3).

Figure 5. Main lobe level as a function of frequency for three similar arrays using different radiators: elastic steel shells, steel shells with material damping (complex Young's modulus), and ideal velocity transducers for comparison.

Figure 6. Resonance effects on directionality: the pattern is shown for four frequencies near resonance.

Figure 7. Frequency dependence of directional pattern of an array of ideal velocity transducers.

Figure 8. Main lobe level as a function of frequency (as in Figure 5) for elastic aluminum shell radiators.

Figure 9. Same as for Figure 4, except that the shells are composed of aluminum rather than steel.

Figure 10. Comparison of directional patterns for point sources, ideal velocity transducers, steel shells, and aluminum shells, for $k a=1$. 